\title{
NOTES ON BOUNDEDNESS OF SPECTRAL MULTIPLIERS ON HARDY SPACES ASSOCIATED TO OPERATORS
}

\author{
BUI THE ANH
}

\begin{abstract}
Let $L$ be a nonnegative self-adjoint operator on $L^{2}(X)$, where $X$ is a space of homogeneous type. Assume that $L$ generates an analytic semigroup $e^{-t L}$ whose kernel satisfies the standard Gaussian upper bounds. We prove that the spectral multiplier $F(L)$ is bounded on $H_{L}^{p}(X)$ for $0<p \leq 1$, the Hardy space associated to operator $L$, when $F$ is a suitable function.
\end{abstract}

\section{$\S 1$. Introduction}

Let $(X, d, \mu)$ be a metric measure space endowed with a distance $d$ and a nonnegative Borel doubling measure $\mu$ on $X$. Recall that the measure $\mu$ satisfies doubling condition if there exists a constant $C>0$ such that, for all $x \in X$ and for all $r>0$,

$$
V(x, 2 r) \leq C V(x, r)<\infty
$$

where $B(x, r)=\{y \in X: d(x, y)<r\}$ and $V(x, r)=\mu(B(x, r))$. In particular, $X$ is a space of homogeneous type. (A more general definition and further studies of these spaces can be found in [CW, chapitre 3].) Note that the doubling property implies the following strong homogeneity property:

$$
V(x, \lambda r) \leq c \lambda^{n} V(x, r)
$$

for some $c, n>0$ uniformly for all $\lambda \geq 1$ and $x \in X$. There also exist $c$ and $N, 0 \leq N \leq n$, such that

$$
V(y, r) \leq c\left(1+\frac{d(x, y)}{r}\right)^{N} V(x, r)
$$

Received July 4, 2010. Revised October 6, 2010. Accepted February 11, 2011.

2010 Mathematics Subject Classification. 34L05, 30H10.

The author's work was partially supported by a Macquarie University scholarship.

(C) 2011 by The Editorial Board of the Nagoya Mathematical Journal 
uniformly for all $x, y \in X$ and $r>0$. Indeed, property (3) with $N=n$ is a direct consequence of the triangle inequality of the metric $d$ and the strong homogeneity property. To simplify notation, we will often use $B$ for $B\left(x_{B}, r_{B}\right)$. Also, given that $\lambda>0$, we will write $\lambda B$ for the $\lambda$-dilated ball, which is the ball with the same center as $B$ and with radius $r_{\lambda B}=\lambda r_{B}$. For each ball $B \subset X$, we set

$$
S_{0}(B)=B \quad \text { and } \quad S_{j}(B)=2^{j} B \backslash 2^{j-1} B \quad \text { for } j \in \mathbb{N} .
$$

In this paper, we assume that $L$ is a nonnegative self-adjoint operator on $L^{2}(X)$ that satisfies the following assumptions.

The operator $L$ generates an analytic semigroup $\left\{e^{-t L}\right\}_{t>0}$ whose kernels $p_{t}(x, y)$ satisfy the Gaussian upper bound; that is, there exist constants $C$, $c>0$ such that, for almost every $x, y \in X$,

$$
\left|p_{t}(x, y)\right| \leq \frac{C}{V(x, \sqrt{t})} \exp \left(-\frac{d^{2}(x, y)}{c t}\right), \quad \forall t>0 .
$$

The Gaussian upper bound considered in [DOS] is more general; that is, there exist constants $C, c>0$ such that, for almost every $x, y \in X$, we have

$$
\left|p_{t}(x, y)\right| \leq \frac{C}{V\left(x, t^{1 / m}\right)} \exp \left(-\frac{d(x, y)^{m /(m-1)}}{c t^{1 /(m-1)}}\right), \quad \forall t>0 .
$$

However, in the case where $m \neq 2$, the results concerning the Hardy spaces in [HLMMY] may not hold. Consequently, in this paper we restrict ourselves to considering the case of $m=2$.

By the spectral theorem, for any bounded Borel function $F:[0, \infty) \rightarrow \mathbb{C}$, one can define the operator

$$
F(L)=\int_{0}^{\infty} F(\lambda) d E(\lambda)
$$

which is bounded on $L^{2}(X)$.

The $L^{p}$-boundedness of spectral multipliers is a well-known problem which has been studied extensively for elliptic operators in [Ho], for sub-Laplacian on nilpotent groups in [C] and [D], for sub-Laplacian on Lie groups of polynomial growth in [A1], for Schrödinger operator on Euclidean space $\mathbb{R}^{n}$ in [He], and for sub-Laplacian on Heisenberg groups in [MSt], among other examples. (For further background information on this topic, we refer the 
reader to $[\mathrm{A} 1],[\mathrm{A} 2],[\mathrm{B}],[\mathrm{C}],[\mathrm{DeM}],[\mathrm{DOS}]$, and $[\mathrm{FS}]$ and the references therein.)

Recently, in [DOS], Duong, Ouhabaz, and Sikora investigated the spectral multiplier theorem in a general setting of abstract operators, which we sketch out briefly here. Let $L$ be a nonnegative self-adjoint operator, and let $L$ generate an analytic semigroup $e^{-t L}$ whose kernel satisfies the standard Gaussian upper bounds (equation (4)). It was proved that if, for $q \in[2, \infty], s>(n / 2)$, and for some $\eta \in C_{c}^{\infty}\left(\mathbb{R}_{+}\right)$,

$$
\sup _{t>0}\left\|\eta \delta_{t} F\right\|_{W_{s}^{q}<\infty}
$$

where $\delta_{t} F(\lambda)=F(t \lambda)$ and $\|F\|_{W_{s}^{q}}=\left\|\left(I-d^{2} / d x^{2}\right)^{s / 2} F\right\|_{L^{q}}$, then $F(L)$ is of weak type $(1,1)$, and hence, by interpolation, $F(L)$ is bounded on $L^{p}(X), 1<$ $p<\infty$.

Working in the same setting as [DOS], this paper is dedicated to studying the boundedness of $F(L)$ when $0<p \leq 1$. We show that $F(L)$ is bounded on $H_{L}^{p}(X)$ for $0<p \leq 1$, the Hardy space associated to the operator $L$. Note that the case when $p=1$ was investigated in [DP] with stronger assumptions imposed on $F$ and $s$. More precisely, it was proved in [DP] that if the nonnegative self-adjoint $L$ satisfies $(G)$, then $F(L)$ is bounded on $H_{L}^{1}(X)$ if (6) holds for $q=\infty$ and $s>n / 2$, or (6) holds for $q=2$ and $s>n / 2+1 / 2$.

The remainder of this article is organized into two sections. In Section 2, we review the definitions and basic properties of Hardy spaces associated to operators in [HLMMY] and [DL]. The main results, Theorem 3.1 and Theorem 3.2, are addressed in Section 3.

\section{$\S 2$. Hardy spaces associated to operators}

The theory of Hardy spaces associated to nonnegative self-adjoint operators satisfying Davies-Gaffney estimates was developed recently by Hofmann, Lu, Mitrea, Mitrea, and Yan [HLMMY]. Here, we use the definitions and characterizations of Hardy spaces $H_{L}^{p}(X)$ from both [HLMMY] and [DL].

\subsection{The atomic Hardy spaces $H_{L}^{p}(X)$ for $p \leq 1$}

Let us describe the notion of a $(p, 2, M)$-atom, $0<p \leq 1$, associated to operators on spaces $(X, d, \mu)$. In what follows, assume that

$$
M \in \mathbb{N} \quad \text { and } \quad M>\frac{n(2-p)}{4 p},
$$


where the parameter $n$ is the constant in (2). Let us denote by $\mathcal{D}(T)$ the domain of an operator $T$.

Definition 2.1.1. A function $a(x) \in L^{2}(X)$ is called a $(p, 2, M)$-atom associated to an operator $L$ if there exist a function $b \in \mathcal{D}\left(L^{M}\right)$ and a ball $B$ of $X$ such that

(i) $a=L^{M} b$;

(ii) $\operatorname{supp} L^{k} b \subset B, k=0,1, \ldots, M$;

(iii) $\left\|\left(r_{B}^{2} L\right)^{k} b\right\|_{L^{2}(X)} \leq r_{B}^{2 M} V(B)^{1 / 2-1 / p}, k=0,1, \ldots, M$.

In the case $\mu(X)<\infty$, the constant function having value $[\mu(X)]^{-1 / p}$ is also considered to be an atom.

Definition 2.1.2. Given $0<p \leq 1$ and $M>n(2-p) / 4 p$, the atomic Hardy space $H_{L, a t, M}^{p}(X)$ is defined as follows. We say that $f=\sum \lambda_{j} a_{j}$ is an atomic $(p, 2, M)$-representation if $\left\{\lambda_{j}\right\}_{j=0}^{\infty} \in l^{p}$, each $a_{j}$ is a $(p, 2, M)$-atom, and the sum converges in $L^{2}(X)$. Set

$$
\mathbb{H}_{L, a t, M}^{p}(X)=\{f: f \text { has an atomic }(p, 2, M) \text {-representation }\}
$$

with the norm given by

$$
\begin{array}{r}
\|f\|_{\mathbb{H}_{L, a t, M}^{p}(X)}=\inf \left\{\left(\sum\left|\lambda_{j}\right|^{p}\right)^{1 / p}: f=\sum \lambda_{j} a_{j}\right. \text { is an atomic } \\
(p, 2, M) \text {-representation }\} .
\end{array}
$$

The space $H_{L, a t, M}^{p}(X)$ is then defined as the completion of $\mathbb{H}_{L, a t, M}^{p}(X)$ with respect to the quasi-metric $d$ defined by $d(h, g)=\|h-g\|_{\mathbb{H}_{L, a t, M}^{p}(X)}$ for all $h, g \in \mathbb{H}_{L, a t, M}^{p}(X)$.

In this case, the mapping $h \rightarrow\|h\|_{H_{L, a t, M}^{p}(X)}, 0<p<1$ is not a norm, and $d(h, g))=\|h-g\|_{H_{L, a t, M}^{p}(X)}$ is a quasi-metric. For $p=1$, the mapping $h \rightarrow\|h\|_{H_{L, a t, M}^{1}(X)}$ is a norm and $H_{L, a t, M}^{1}(X)$ is complete. In particular, $H_{L, a t, M}^{1}(X)$ is a Banach space and $H_{L, a t, M}^{1}(X) \hookrightarrow L^{1}$. A basic result concerning these spaces is the following proposition.

Proposition 2.1.3. If a nonnegative self-adjoint operator $L$ satisfies $(G)$, then for every $0<p \leq 1$ and for all integers $M \in \mathbb{N}$ with $M>(n(2-p) / 4 p$, the spaces $H_{L, a t, M}^{p}(X)$ coincide and their norms are equivalent. 
For the proof, we refer to [HLMMY, Theorem 5.1] for $p=1$ and to [DL, Section 3] for $p<1$.

We next describe the notion of a $(p, 2, M, \epsilon)$-molecule associated to an operator $L$.

Definition 2.1.4. Let $0<p \leq 1$, let $0<\epsilon$, and let $M \in \mathbb{N}$. A function $\alpha \in L^{2}(X)$ is called a $(p, 2, M, \epsilon)$-molecule associated to $L$ if there exist a function $b \in D\left(L^{M}\right)$ and a ball $B$ such that

(i) $\alpha=L^{M} b$;

(ii) for every $k=0,1, \ldots, M$ and $j=0,1, \ldots$, there holds

$$
\left\|\left(r_{B}^{2} L\right)^{k} b\right\|_{L^{2}\left(S_{j}(B)\right)} \leq r_{B}^{2 M} 2^{-j \epsilon} V\left(2^{j} B\right)^{1 / 2-1 / p} .
$$

Proposition 2.1.5. Suppose that $0<p \leq 1$ and that $M>(n(2-p) / 4 p)$. If $\alpha$ is a $(p, 2, M, \epsilon)$-molecule or an $(p, 2, M)$-atom associated to $L$, then $\alpha \in H_{L}^{p}(X)$. Moreover, $\|\alpha\|_{H_{L}^{p}(X)}$ is independent of $M$.

For the proof, we refer the reader to [HLMMY] for $p=1$ and to [DL] for $p<1$.

\subsection{A characterization of $H_{L, a t, M}^{p}(X)$ in terms of square functions}

Define

$$
S_{h} f(x)=\left(\int_{0}^{\infty} \int_{d(x, y)<t}\left|t^{2} L e^{-t^{2} L} f(y)\right|^{2} \frac{d \mu(y)}{V(x, t)} \frac{d t}{t}\right)^{1 / 2}, \quad x \in X
$$

The space $H_{L, S_{h}}^{p}(X)$ is defined as the completion of

$$
\left\{f \in L^{2}(X):\left\|S_{h} f\right\|_{L^{p}(X)}<\infty\right\}
$$

under the norm given by the $L^{p}$-norm of the square function; that is,

$$
\|f\|_{H_{L, S_{h}}^{p}(X)}=\left\|S_{h} f\right\|_{L^{p}(X)}, \quad 0<p \leq 1 .
$$

Then the square function and atomic $H^{p}$-spaces are equivalent, if the parameter $M>n(2-p) / 4 p$. In fact, we have the following result.

Proposition 2.2.1. Suppose that $0<p \leq 1$ and that $M>n(2-p) / 4 p$. Then we have $H_{L, a t, M}^{p}=H_{L, S_{h}}^{p}(X)$, and their norms are equivalent.

Proof. For the proof, see [DL, Theorem 3.12]. 
Consequently, as in Definition 2.2.2, one may write $H_{L, a t}^{p}$ in place of $H_{L, a t, M}^{p}$ when $M>n(2-p) / 4 p$. Precisely, we have the following definition.

Definition 2.2.2. The Hardy space $H_{L}^{p}(X), p \geq 1$, is the space

$$
H_{L}^{p}(X):=H_{L, S_{h}}^{p}(X):=H_{L, a t}^{p}(X):=H_{L, a t, M}^{p}(X), \quad M>\frac{n(2-p)}{4 p} .
$$

We end this section with the following result, which plays an important role in the remainder of this article.

Proposition 2.2.3. Let $T$ be a bounded linear operator on $L^{2}(X)$. If there exists $C_{0}>0$ such that for any $(p, 2, M)$-atom $a, 0<p \leq 1$, one has

$$
\|T a\|_{H_{L}^{p}(X)} \leq C_{0}
$$

then $T$ can be extended to a bounded operator on $H_{L}^{p}(X)$; moreover, there exists $\kappa>0$ so that $\|T\|_{H_{L}^{p}(X) \rightarrow H_{L}^{p}(X)} \leq \kappa C_{0}$.

The proof is similar to one in [HM, Lemma 4.1], so we omit details here.

\section{§3. Spectral multiplier theorem on $H_{L}^{p}(X), 0<p \leq 1$}

Let $T$ be a bounded linear operator on $L^{2}(X)$. Let the associated kernel to the operator $T$ be denoted by $K_{T}(x, y)$. By the kernel $K_{T}(x, y)$, we mean

$$
T f(x)=\int_{X} K_{T}(x, y) f(y) d \mu(y),
$$

where $K_{T}(x, y)$ is a measurable function and the formula above holds for each continuous function $f$ with compact support and for almost all $x$ not in the support of $f$.

Our main results are the following two theorems.

THEOREM 3.1. Let $L$ be a nonnegative self-adjoint operator satisfying $(G)$. Suppose that $s>n(2-p) / 2 p$, and suppose that, for any $R>0$ and for all Borel functions $F$ such that $\operatorname{supp} F \subset[0, R]$,

$$
\int_{X}\left|K_{F(\sqrt{L})}(x, y)\right|^{2} d \mu(x) \leq \frac{C}{V\left(y, R^{-1}\right)}\left\|\delta_{R} F\right\|_{L^{q}}^{2}
$$

for some $q \in[2, \infty]$. Then for any Borel function $F$ such that $\sup _{t>0}\left\|\eta \delta_{t} F\right\|_{W_{s}^{q}}<\infty$, the operator $F(L)$ is bounded on $H_{L}^{p}(X)$ for all $0<p \leq 1$. 
Note that (8) always holds for $q=\infty$ (see [DOS]). If (8) holds for some $q<\infty$, then the pointwise spectrum of $L$ is empty. Indeed, for all $p<\infty$ and all $y \in X$, we have

$$
0=C\left\|\delta_{R} \chi_{\{a\}}\right\|_{L^{q}} \leq V(y, 1 / R)^{1 / 2}\left\|K_{\chi_{\{a\}}(\sqrt{L})}(\cdot, y)\right\|_{L^{2}},
$$

so $\chi_{\{a\}}(\sqrt{L})=0$. Hence, for elliptic operators on compact manifolds, (8) cannot be true for any $q<\infty$. To be able to study these operators as well, we introduce some variation of condition (8). Following [CS] and [DOS] for a Borel function $F$ such that $\operatorname{supp} F \subset[-1,2]$, we define the norm $\|F\|_{N, q}$ by the formula

$$
\|F\|_{N, q}=\left(\frac{1}{3 N} \sum_{l=1-N}^{2 N} \sup _{\lambda \in\left[\frac{l-1}{N}, \frac{l}{N}\right)}|F(\lambda)|^{q}\right)^{1 / q},
$$

where $q \in[1, \infty)$ and $N \in \mathbb{Z}_{+}$. For $q=\infty$, we put $\|F\|_{N, q}=\|F\|_{L^{\infty}}$. It is obvious that $\|F\|_{N, q}$ increases monotonically in $q$. The next theorem is a variation of Theorem 3.1. This variation can be used in case of operators with nonempty pointwise spectrum (see [CS, Theorem 3.6]).

Theorem 3.2. Assume that $\mu(X)<\infty$. Let $L$ be a nonnegative selfadjoint operator satisfying $(G)$. Suppose that $s>n / 2$ and for any $N \in \mathbb{Z}_{+}$ and all Borel functions $F$ such that $\operatorname{supp} F \subset[-1, N+1]$,

$$
\int_{X}\left|K_{F(\sqrt{L})}(x, y)\right|^{2} d \mu(x) \leq \frac{C}{V(y, 1 / N)}\left\|\delta_{N} F\right\|_{N, q}^{2}
$$

for some $q \in[2, \infty]$. Then for any Borel function $F$ such that $\sup _{t>0}\left\|\eta \delta_{t} F\right\|_{W_{s}^{q}}<\infty$, the operator $F(L)$ is bounded on $H_{L}^{1}(X)$.

(For further discussion on conditions (8) and (9), we refer the reader to [DOS, pp. 467-480]).

Remark 3.3. In Theorem 3.1, we can extend $F(L)$ to a bounded operator on $H_{L}^{p}(X)$ for all $0<p \leq 1$, whereas Theorem 3.2 only establishes the boundedness of $F(L)$ on $H_{L}^{1}(X)$. This is a reason why in Theorem 3.2 we require $s>n / 2$ instead of $s>n(2-p) / 2 p$ as in Theorem 3.1.

In both Theorems 3.1 and 3.2 , the kernel $K_{F(\sqrt{L})}(x, y)$ of $F(\sqrt{L})$ always exists. Indeed, in virtue of the Fourier inversion formula

$$
G\left(L / R^{2}\right) e^{-L / R^{2}}=\frac{1}{2 \pi} \int_{\mathbb{R}} \exp \left((i \tau-1) R^{-2} L\right) \widehat{G}(\tau) d \tau,
$$


and so

$$
K_{F(\sqrt{L})}(x, y)=\frac{1}{2 \pi} \int_{\mathbb{R}} \widehat{G}(\tau) p_{(i \tau-1) R^{-2}}(x, y) d \tau
$$

where $G(\lambda)=\left[\delta_{R} F\right](\sqrt{L}) e^{\lambda}$. (For details, we refer the reader to [DOS, p. 454].)

As a preamble to the proof of Theorems 3.1 and 3.2, we record a useful auxiliary result, which is taken from [DOS, Lemma 4.3].

LEMMA 3.4. Let $L$ be a nonnegative self-adjoint operator satisfying $(G)$.

(a) If $L$ satisfies (8) for some $q \in[2, \infty], R>0$ and $s>0$, then for any $\epsilon>0$, there exists a constant $C=C(s, \epsilon)$ such that

(10) $\int_{X}\left|K_{F(\sqrt{L})}(x, y)\right|^{2}(1+R d(x, y))^{s} d \mu(x) \leq \frac{C}{V\left(y, R^{-1}\right)}\left\|\delta_{R} F\right\|_{W_{\frac{s}{2}+\epsilon}^{q}}^{2}$

for all Borel functions $F$ such that $\operatorname{supp} F \subseteq[R / 4, R]$.

(b) If $L$ satisfies (9) for some $q \in[2, \infty]$ and if $N>8$ is a natural number, then for any $s>0, \epsilon>0$, and function $\xi \in C_{c}^{\infty}([-1,1])$, there exists a constant $C=C(s, \epsilon, \xi)$ such that

(11) $\quad \int_{X}\left|K_{F * \xi(\sqrt{L})}(x, y)\right|^{2}(1+N d(x, y))^{s} d \mu(x) \leq \frac{C}{V\left(y, R^{-1}\right)}\left\|\delta_{N} F\right\|_{W_{\frac{s}{2}+\epsilon}^{q}}^{2}$

for all Borel functions $F$ such that $\operatorname{supp} F \subseteq[N / 4, N]$.

Proof of Theorem 3.1. Since condition $\sup _{t>0}\left\|\eta \delta_{t} F\right\|_{W_{s}^{q}}<\infty$ is invariant under the change of variable $\lambda \mapsto \sqrt{\lambda}$ and independent on the choice of $\eta$, the $H_{L}^{p}(X)$-boundedness of $F(L)$ and $F(\sqrt{L})$ is equivalent. Hence, instead of proving the $H_{L}^{p}(X)$-boundedness of $F(L)$, we will show that $F(\sqrt{L})$ is bounded on $H_{L}^{p}(X)$. Due to Proposition 2.2.3, it suffices to show that there exists $\epsilon>0$ such that, for any $(p, 2,2 M)$-atom $a=L^{2 M} b$ in $H_{L}^{p}$, the function

$$
\widetilde{a}=F(\sqrt{L}) a
$$

is a multiple of a $(p, 2, M, \epsilon)$-molecule for $M>n(2-p) / 4 p$.

By standard argument, fix a function $\phi \in C_{c}^{\infty}(1 / 4,1)$ such that

$$
\sum_{j \in \mathbb{Z}} \phi\left(2^{-j} \lambda\right)=1 \quad \text { for } \lambda>0 \text {. }
$$


Set $j_{0}=-\log _{2} r_{B}$. Then, for $0 \leq k \leq M$, one has

$$
\begin{aligned}
\left(r_{B}^{2} L\right)^{k} \widetilde{b}= & r_{B}^{2 k} \sum_{j \geq j_{0}} \phi\left(2^{-j} \sqrt{L}\right) F(\sqrt{L}) L^{k+M} b \\
& +r_{B}^{2 k} \sum_{j<j_{0}} \phi\left(2^{-j} \sqrt{L}\right) L^{M} F(\sqrt{L}) L^{k} b \\
= & r_{B}^{2 k} \sum_{j \geq j_{0}} \phi\left(2^{-j} \sqrt{L}\right) F(\sqrt{L}) b_{1}+r_{B}^{2 k} \sum_{j<j_{0}} \phi\left(2^{-j} \sqrt{L}\right) L^{M} F(\sqrt{L}) b_{2}
\end{aligned}
$$

where $\widetilde{b}=L^{M} b$.

It is easy to see that

$$
\left\|b_{1}\right\|_{L^{2}} \leq r_{B}^{2 M-2 k} V(B)^{\frac{1}{2}-\frac{1}{p}} \quad \text { and } \quad\left\|b_{2}\right\|_{L^{2}} \leq r_{B}^{4 M-2 k} V(B)^{\frac{1}{2}-\frac{1}{p}}
$$

Setting

$$
F_{j}(\lambda)= \begin{cases}F(\lambda) \phi\left(2^{-j} \lambda\right), & j \geq j_{0} \\ F(\lambda)\left(2^{-j} \lambda\right)^{2 M} \phi\left(2^{-j} \lambda\right), & j<j_{0},\end{cases}
$$

then we can rewrite (12) as follows

$$
\left(r_{B}^{2} L\right)^{k} \widetilde{b}=r_{B}^{2 k} \sum_{j \geq j_{0}} F_{j}(\sqrt{L}) b_{1}+r_{B}^{2 k} 2^{2 j M} \sum_{j<j_{0}} F_{j}(\sqrt{L}) b_{2} .
$$

Since (13) converges in $L^{2}(X)$, we have, for any $k \geq 0$,

$$
\begin{aligned}
\left\|\left(r_{B}^{2} L\right)^{k} \widetilde{b}\right\|_{L^{2}\left(S_{k}(B)\right) \leq} & r_{B}^{2 k} \sum_{j \geq j_{0}}\left\|F_{j}(\sqrt{L}) b_{1}\right\|_{L^{2}\left(S_{k}(B)\right)} \\
& +r_{B}^{2 k} 2^{2 j M} \sum_{j<j_{0}}\left\|F_{j}(\sqrt{L}) b_{2}\right\|_{L^{2}\left(S_{k}(B)\right)} .
\end{aligned}
$$

First, let us estimate $\left\|F_{j}(\sqrt{L}) b_{1}\right\|_{L^{2}\left(S_{k}(B)\right)}$ for $j \geq j_{0}$. Since $\operatorname{supp} F_{j} \subset$ $[R / 4, R]$ with $R=2^{j}$, by applying Lemma 3.4 and the Minskowski inequality, we have, for $s>s^{\prime}>n(2-p) / 2 p \geq n / 2$ and $k \geq 2$,

$$
\begin{aligned}
& \left\|F_{j}(\sqrt{L}) b_{1}\right\|_{L^{2}\left(S_{k}(B)\right)} \\
& \quad \leq\left\|\int_{B} K_{F_{j}(\sqrt{L})}(x, y) b_{1}(y) d \mu(y)\right\|_{L^{2}\left(S_{k}(B)\right)} \\
& \quad \leq\left\|b_{1}\right\|_{L^{1}} \sup _{y \in B}\left(\int_{S_{k}(B)}\left|K_{F_{j}(\sqrt{L})}(x, y)\right|^{2} d \mu(x)\right)^{1 / 2}
\end{aligned}
$$




$$
\begin{aligned}
\leq & \left\|b_{1}\right\|_{L^{2}} V(B)^{\frac{1}{2}} \sup _{y \in B}\left(\int_{S_{k}(B)}\left|K_{F_{j}(\sqrt{L})}(x, y)\right|^{2} d \mu(x)\right)^{1 / 2} \\
\leq & r_{B}^{2 M-2 k} V(B)^{1-\frac{1}{p}}\left(2^{-(j+k) s^{\prime}} r_{B}^{s^{\prime}}\right) \\
& \times \sup _{y \in B}\left(\int_{S_{k}(B)}\left|K_{F_{j}(\sqrt{L})}(x, y)\right|^{2}\left(1+2^{j} d(x, y)\right)^{2 s^{\prime}} d \mu(x)\right)^{1 / 2} \\
\leq & C r_{B}^{2 M-2 k} V(B)^{1-\frac{1}{p}}\left(2^{-(j+k) s^{\prime}} r_{B}^{s^{\prime}}\right) \sup _{y \in B} \frac{1}{\sqrt{V\left(y, 2^{-j}\right)}}\left\|\delta_{2^{j}} F_{j}\right\|_{W_{s}^{q}} \\
\leq & C r_{B}^{2 M-2 k} V(B)^{1-\frac{1}{p}}\left(2^{-(j+k) s^{\prime}} r_{B}^{s^{\prime}}\right) \sup _{y \in B} \frac{1}{\sqrt{V\left(y, 2^{-j}\right)}} .
\end{aligned}
$$

For $j \geq j_{0}=-\log _{2} r_{B}$, we have, by (3),

$$
\sup _{y \in B} \frac{1}{V\left(y, 2^{-j}\right)}=\sup _{y \in B} \frac{1}{V\left(y, r_{B} 2^{j_{0}-j}\right)} \leq C \sup _{y \in B} \frac{\left(2^{j} r_{B}\right)^{n}}{V\left(y, r_{B}\right)} \leq C \frac{\left(2^{j} r_{B}\right)^{n}}{V(B)} .
$$

This together with (14) yields

$$
\begin{aligned}
\left\|F_{j}(\sqrt{L}) b_{1}\right\|_{L^{2}\left(S_{k}(B)\right)} & \leq C r_{B}^{2 M-2 k} V(B)^{1-\frac{1}{p}} 2^{-(j+k) s^{\prime}} 2^{-s^{\prime} j_{0}} \frac{\left(2^{j} r_{B}\right)^{\frac{n}{2}}}{V(B)^{\frac{1}{2}}} \\
& \leq C r_{B}^{2 M-2 k} V\left(2^{k} B\right)^{\frac{1}{2}-\frac{1}{p}} 2^{-k\left(s^{\prime}-\frac{n(2-p)}{2 p}\right)} 2^{\left(j-j_{0}\right)\left(\frac{n}{2}\right)-s^{\prime}} .
\end{aligned}
$$

For $k=0,1$, it is not difficult to see that

$$
\left\|F_{j}(\sqrt{L}) b_{1}\right\|_{L^{2}\left(S_{k}(B)\right)} \leq\left\|b_{1}\right\|_{L^{2}\left(S_{k}(B)\right)} \leq C r_{B}^{2 M-2 k} 2^{-k \epsilon} V\left(2^{k} B\right)^{\frac{1}{2}-\frac{1}{p}},
$$

with $\epsilon=s^{\prime}-n(2-p) / 2 p$.

Therefore,

$$
r_{B}^{2 k} \sum_{j \geq j_{0}}\left\|F_{j}(\sqrt{L}) b_{1}\right\|_{L^{2}\left(S_{k}(B)\right)} \leq C 2^{-k \epsilon} r_{B}^{2 M} V\left(2^{k} B\right)^{\frac{1}{2}-\frac{1}{p}} .
$$

Note that for $j \leq j_{0}$,

$$
\sup _{y \in B} \frac{1}{V\left(y, 2^{-j}\right)}=\sup _{y \in B} \leq C \frac{1}{V\left(y, r_{B} 2^{j_{0}-j}\right)} \leq \sup _{y \in B} C \frac{1}{V\left(y, r_{B}\right)}=\frac{C}{V(B)} .
$$

At this stage, repeating the argument above, we also obtain

$$
r_{B}^{2 k} 2^{2 j M} \sum_{j<j_{0}}\left\|F_{j}(\sqrt{L}) b_{2}\right\|_{L^{2}\left(S_{k}(B)\right)} \leq C 2^{-k \epsilon} r_{B}^{2 M} V\left(2^{k} B\right)^{\frac{1}{2}-\frac{1}{p}} .
$$


Hence, $\widetilde{a}=F(\sqrt{L}) a$ is a multiple of a $(p, 2, M, \epsilon)$-molecule. The proof is complete.

Proof of Theorem 3.2. First, we claim that if $F$ supported in $[-1, N+1]$ satisfies (9), then

$$
\|F(\sqrt{L})\|_{H_{L}^{1} \rightarrow H_{L}^{1}}^{2} \leq C N^{n}\left\|\delta_{N} F\right\|_{N, q} .
$$

Since $\mu(X)<\infty, X$ is bounded. Therefore, there exists $r_{0}>1$ such that $X \subset B\left(z, r_{0}\right)$ for all $z \in X$.

Let $a=L^{M} b$ be a $(1,2, M)$-atom associated to some ball $B$. We will show that $F(\sqrt{L}) a=L^{M} F(\sqrt{L}) b$ is a multiple of $(1,2, M)$-atom associated to the ball $B(z, \gamma)$ for all $z \in X$ and $\gamma=\max \left\{r_{B}, r_{0}\right\}$. Indeed, by Minskowski inequality, we have, for all $0 \leq k \leq M$,

$$
\begin{aligned}
\left\|L^{k} F(\sqrt{L}) b\right\|_{L^{2}(B(z, \gamma))}^{2} & =\left\|F(\sqrt{L})\left(L^{k} b\right)\right\|_{L^{2}(B(z, \gamma))}^{2} \\
& =\left\|\int_{X} K_{F(\sqrt{L})}(x, y)\left(L^{k} b\right)(y) d \mu(y)\right\|_{L^{2}(X)}^{2} \\
& \leq\left(\int_{X}\left\|K_{F(\sqrt{L})}(\cdot, y)\right\|_{L^{2}}\left|\left(L^{k} b\right)(y)\right| d \mu(y)\right)^{2} .
\end{aligned}
$$

Since $a$ is a $(1,2, M)$-atom,

$$
\int_{X}\left|\left(L^{k} b\right)(y)\right| d \mu(y) \leq V(B)^{-1 / 2}\left\|L^{k} b\right\|_{L^{2}(B)} \leq r_{B}^{2 M-2 k} .
$$

So, we get

$$
\begin{aligned}
\left\|L^{k} F(\sqrt{L}) b\right\|_{L^{2}(B(z, \gamma))}^{2} & \leq C \frac{r_{B}^{4 M-4 k}}{V(y, 1 / N)}\left\|\delta_{N} F\right\|_{N, q}^{2} \\
& \leq C \frac{\left(r_{0} N\right)^{n}}{V\left(y, r_{0}\right)} r_{B}^{4 M-4 k}\left\|\delta_{N} F\right\|_{N, q}^{2} \\
& \leq \frac{C}{V(z, \gamma)} \gamma^{4 M-4 k} N^{n}\left\|\delta_{N} F\right\|_{N, q}^{2}
\end{aligned}
$$

Hence, $F(\sqrt{L}) a$ is a multiple of $(1,2, M)$-atom associated to the ball $B(z, \gamma)$ for any $z \in X$ with a constant $N^{n / 2}\left\|\delta_{N} F\right\|_{N, q}$. Therefore, due to Proposition 2.1.5, one has $\|F(\sqrt{L}) a\|_{H_{L}^{1}}^{2} \leq C N^{n}\left\|\delta_{N} F\right\|_{N, q}^{2}$. So, Proposition 2.2.3 tells us that

$$
\|F(\sqrt{L})\|_{H_{L}^{1} \rightarrow H_{L}^{1}}^{2} \leq C N^{n}\left\|\delta_{N} F\right\|_{N, q}^{2}
$$


Therefore, in order to prove Theorem 3.2, we can assume that $\operatorname{supp} F \subset$ $[1, \infty]$. Let $\phi$ be the function as in the proof of Theorem 3.1. We set $F^{k}(\lambda)=$ $\phi\left(2^{-k} \lambda\right) F(\lambda)$, and

$$
\tilde{F}=\sum_{k=1}^{\infty} F^{k} * \xi
$$

where $\xi$ is a function defined in (b) of Lemma 3.4.

By repeating the proof of Theorem 3.1 and using (9) in place of (8), we can prove that the $\tilde{F}(\sqrt{L})$ is bounded on $H_{L}^{1}(X)$. Hence, it suffices to show that $F(\sqrt{L})-\tilde{F}(\sqrt{L})$ is bounded on $H_{L}^{1}(X)$. To do this, we write

$$
F-\tilde{F}=\sum_{k} H_{k}, \quad \text { where } H_{k}=F^{k}-F^{k} * \xi .
$$

Since $\operatorname{supp} H_{k} \subset\left[-1,2^{k}+1\right]$, due to (15), we have

$$
\left\|H_{k}(\sqrt{L})\right\|_{H_{L}^{1} \rightarrow H_{L}^{L}} \leq C 2^{k n}\left\|\delta_{2^{k}} H_{k}\right\|_{2^{k}, q} .
$$

Therefore, to complete our proof, we need only to show that $\sum_{k} 2^{k n}\left\|\delta_{2^{k}} H_{k}\right\|_{2^{k}, q}$. To do this, we make the following claim (see [DOS, Proposition 4.6]).

Proposition 3.5. Suppose that $\xi \in C_{c}^{\infty}$ is a function such that $\operatorname{supp} \xi \subset$ $[-1,1], \xi \geq 0, \widehat{\xi}(0)=1$ and $\widehat{\xi}^{(k)}(0)=0$ for all $1 \leq k \leq[s]+2$. If $\operatorname{supp} G \subset$ $[0,1]$, then

$$
\left\|G-G * \xi_{N}\right\|_{N, q} \leq C N^{-s}\|G\|_{W_{s}^{q}}
$$

for all $s>1 / q$.

In virtue of Proposition 3.5, we have

$$
\begin{aligned}
\sum_{k} 2^{k n}\left\|\delta_{2^{k}} H_{k}\right\|_{2^{k}, q} & =\sum_{k} 2^{k n}\left\|\delta_{2^{k}}\left[F^{k}\right]-\xi_{2^{k}} * \delta_{2^{k}}\left[F^{k}\right]\right\|_{2^{k}, q} \\
& \leq C \sum_{k} 2^{n k} 2^{-2 k s}\left\|\delta_{2^{k}}\left[F^{k}\right]\right\|_{W_{s}^{q}}^{2} \\
& \leq C \sup _{k>0}\left\|\delta_{2^{k}}\left[F^{k}\right]\right\|_{W_{s}^{q}}^{2},
\end{aligned}
$$

where $\xi_{2^{k}}$ denotes the function $\xi\left(2^{-k} \cdot\right)$.

This completes our proof. 
Acknowledgments. This paper is part of the author's Ph.D. dissertation, and he thanks his supervisor, Prof. X. T. Duong, for helpful comments and suggestions. The author is also very grateful to the referees for helpful comments and advice.

\section{REFERENCES}

[A1] G. Alexopoulos, Spectral multipliers on Lie groups of polynomial growth, Proc. Amer. Math. Soc. 120 (1994), 973-979.

[A2] - Spectral multipliers for Markov chains, J. Math. Soc. Japan 56 (2004), 833-852.

[B] S. Blunck, A Hörmander-type spectral multiplier theorem for operators without heat kernel, Ann. Sc. Norm. Super. Pisa Cl. Sci. (5) 2 (2003), 449-459.

[C] M. Christ, $L^{p}$ bounds for spectral multipliers on nilpotent groups, Trans. Amer. Math. Soc. 328 (1991), 73-81.

[CS] M. Cowling and A. Sikora, A spectral multiplier theorem for a sublaplacian on SU(2), Math. Z. 238 (2001), 1-36.

[CW] R. Coifman and G. Weiss, Analyse harmonique non-commutative sur certains espaces homogènes, Lecture Notes in Math. 242, Springer, Berlin, 1971.

[DeM] L. De Michele and G. Mauceri, $H^{p}$ multpliers on stratified groups, Ann. Mat. Pura Appl. 148 (1987), 353-366.

[D] X. T. Duong, From the $L^{1}$ norms of the complex heat kernels to a Hörmander multiplier theorem for sub-Laplacians on nipotent Lie groups, Pacific J. Math. 173 (1996), 413-424.

[DL] X. T. Duong and J. Li, Hardy spaces associated to operators satisfying bounded $H_{\infty}$ functional calculus and Davies-Gaffney estimates, preprint, 2009.

[DOS] X. T. Duong, E. M. Ouhabaz, and A. Sikora, Plancherel-type estimates and sharp spectral multipliers, J. Funct. Anal. 196 (2002), 443-485.

[DP] J. Dziubański and M. Preisner, Remarks on spectral multiplier theorems on Hardy spaces associated with semigroups of operators, Rev. Un. Mat. Argentina 50 (2009), 201-215.

[FS] G. B. Folland and E. M. Stein, Hardy Spaces on Homogeneous Groups, Math. Notes 28, Princeton University Press, Princeton, 1982.

[He] W. Hebisch, A multiplier theorem for Schrödinger operators, Colloq. Math. 60/61 (1990), 659-664.

[HLMMY] S. Hofmann, G. Lu, D. Mitrea, M. Mitrea, and L. Yan, Hardy spaces associated to nonnegative self-adjoint operators satisfying Davies-Gaffney estimates, preprint http://www.ams.org/journals/memo/0000-000-00/ (accessed 7 July 2011).

[HM] S. Hofmann and S. Mayboroda, Hardy and BMO spaces associated to divergence form elliptic operators, Math. Ann. 344 (2009), 37-116.

[Ho] L. Hörmander, The spectral function of an elliptic operator, Acta Math. 121 (1968), 193-218. 
[MSt] D. Müller and E. M. Stein, On spectral multipliers for Heisenberg and related groups, J. Math. Pures Appl. 73 (1994), 413-440.

Department of Mathematics

University of Pedagogy

HoChiMinh City

Vietnam

bt_anh80@yahoo.com

Current:

Department of Mathematics

Macquarie University

NSW 2109, Australia

the.bui@mq.edu. au 